\title{
Research on Incentive and Restraint Mechanisms for the Construction of Local Government Affordable Housing
}

\author{
Xue Tan \\ School of Finance \\ Harbin University of Commerce \\ Harbin, China \\ 12530731@qq.com
}

\begin{abstract}
In China, execution of the construction of affordable housing is mainly relied on the actively cooperation by local governments. Local government plays a key role in the financing process of affordable housing. How to actively pursue the construction of affordable housing and the successful complete the task under the situation that the revenue of local government is decreasing and the fiscal spending is increasing, is the problem which needs to be solved by the central government. In this paper, for solving this problem, we use game theory to build the model and design the mechanism, to provide policy recommendations for the central government. Problems faced by the central government are to determine a feasible incentive mechanism, and makes its expected revenue $E u_{1}$ to be maximized. Central government confirms the reward system $(\underline{r}, \underline{h})$ and $(r$, $h$ )based on local government' judgment rate to mobilize enthusiasm and initiative of local government on construct affordable housing.
\end{abstract}

Keywords-affordable housing; local government; incentive and restraint

\section{INTRODUCTION}

From the start of the construction of affordable housing plan, local governments actively cooperate it and finish their task properly. In the process of completion of affordable housing by local governments, they mainly through the land transfer income and financial allocation to make the financing for affordable housing. Construction of affordable housing is related to the colossal task; it is directly related to the housing problems of low-income populations; it determines whether living standards of people are improved or not, thus the central government attaches great importance on it.

However, in the process of construction of affordable housing, the main role of the central government is policymaking and guidance. In particular execution, it still relies on local governments' actively cooperation and hardworking. Local governments need to work closely with the decisions made by the central government, play a key role in the financing of affordable housing. Local governments not only need to take a part of their own revenue for the financial expenditures, but also needs to rely on a variety of local

Supported by the Heilongjiang Social Science Foundation (16JYE13) and the Young Innovative Talents Project of Harbin University of Commerce (2016QN022) financing platforms to complete the financing of affordable housing according to the specific circumstances of each place.

It is well known that the financing is the most difficult problem in the construction of affordable housing, and this problem is primarily responsible for the local government. For local governments, to complete the task of building affordable housing, not only means less revenue, but also means an increasing in fiscal spending. How can enhance the enthusiasm of local governments to build affordable housing, under the situation of decreasing in revenue and increasing in fiscal spending, actively implementing the construction of affordable housing is an issue that central government must face. In this paper, we focus on the subject of how to enhance the enthusiasm of local governments to build affordable housing by the central government, using game theory to build the model and design the mechanism, to provide policy recommendations for the central government.

\section{MECHANISM DESIGN FOR LOCAL GOVERNMENT'S CONSTRUCTION OF AFFORDABLE HOUSING}

\section{A. The basic assumption on the central government}

The central government in order to encourage local governments to complete the task of building affordable housing, usually use performance assessment to measure the situation of task completion. If they can complete tasks assigned by the central government excellently, the central government will give local governments some incentives, which will be some specific policy preferences and policy supports. Assume that the task central government issued for construction of affordable housing is $r$ sets, central government's gains from it after the completion is $T(r)$, specific performance of gains is that it can solve the housing problems of low-income groups, thus make contribution to social stability and economic development.

The central government needs to give certain incentives to local governments, and needs to pay a certain cost, design cost function $h(r)$, the size of the cost is proportional to the number of affordable housing construction, set this function to be a for common knowledge both buyers and sellers.

The central government's profit function should be: 


$$
u_{1}(r)=T(r)-h(r)
$$

\section{B. The basic assumption on local governments}

After local governments finished the task $r$ of affordable housing construction by central government, its benefits from central government should be $h(r)$. Its financing for the construction of affordable housing, the construction cost is set to be $\theta s(r)$. The function $s(r)$ is the common knowledge of both buyers and sellers, $\theta$ is the local government's private information, securities companies are not known. Thus, the function for the profitability of local government should be:

$$
u_{2}(r)=\theta h(r)-s(r)
$$

\section{Model building}

Set the enthusiasm of completion of affordable housing by the local government is divided into two different types, that $\Theta=\{\bar{\theta}, \underline{\theta}\}$, in which $\bar{\theta}>\underline{\theta}>0$. This is unknown by the central government, but the central government can get the inference of the type of local governments, that the proportion of $\bar{\theta}$ is $p$, so the proportion of $\underline{\theta}$ is $1-p$. For the central government, because do not know exactly which type the local government belongs to, is $\bar{\theta}$ or $\underline{\theta}$, therefore it must design two sets of performance incentive programs: use $(\underline{r}, \underline{h})$ for investors with $\underline{\theta}$ type, use $(\bar{r}, \bar{h})$ for investors with $\bar{\theta}$ type. Expected revenue function for the central government should be:

$$
E u_{1}=p(\bar{T}-\bar{h})+(1-p)(\underline{T}-\underline{h})
$$

Local governments will face constraints from two sides. One is the individual rationality constraint $(I R)$, this is the basic condition that government willing to participate in the game, otherwise the local government is not willing to complete the task of building affordable housing. Two programs should be designed for the central government, there should be:

$$
\begin{gathered}
I R_{1}: \underline{\theta} h(\underline{r})-s(\underline{r}) \geq 0 \\
I R_{2}: \bar{\theta} h(\bar{r})-s(\bar{r}) \geq 0
\end{gathered}
$$

The other constraint is the constraint of incentive compatibility constraint $(I C)$. That is, in the situation that the central government does not know the type of the local government, the local government under the scheme designed by central government to be motivated to select the action desired by the central government. This requires that when local governments select to do the action, they will have better benefits than they do not take any actions. For two options of the central government, there should be:

$$
\begin{aligned}
& I C_{1}: \underline{\theta} h(\underline{r})-s(\underline{r}) \geq \underline{\theta} h(\bar{r})-s(\bar{r}) \\
& I C_{2}: \bar{\theta} h(\bar{r})-s(\bar{r}) \geq \bar{\theta} h(\underline{r})-s(\underline{r})
\end{aligned}
$$

If the central government's incentives while satisfying the constraints $I R$ and $I C$, then this is feasible. The ultimate goal of central government is to achieve the maximum benefits, i.e. in four constraints of IR and IC to find the optimal solution of the equation (3). Problems faced by the central government are to determine a feasible incentive mechanism, and makes its expected revenue $E u_{l}$ to be maximized.

\section{MODEL SOLUTION}

Assuming reward systems“" $(\underline{r}, \underline{h})$ and $(\bar{r}, \bar{h})$ ”as feasible alternative, they must simultaneously meet the four constraint conditions for $I R$ and $I C$ and enable $E u_{l}$ to be maximized.

\section{A. If IRI and $I C_{2}$ are true, then IR2 become true automatically}

By $I R_{l}$, it can be known $s(\underline{r}) \leq \underline{\theta} h(\underline{r})$, by $I C_{2}$, it can be known $\bar{\theta} h(\bar{r})-s(\bar{r}) \geq \bar{\theta} h(\underline{r})-s(\underline{r})$, and then:

$$
\bar{\theta} h(\bar{r})-s(\bar{r}) \geq \bar{\theta} h(\underline{r})-s(\underline{r}) \geq(\bar{\theta}-\underline{\theta}) h(\underline{r}) \geq 0,
$$

then $\bar{\theta} h(\bar{r})-s(\bar{r}) \geq 0$.

That is constraint for $I R_{2}$ is true, the original four constraint conditions are reduced to three. Unless $\underline{r}=0, h(r)$ is not likely to be 0 , at the same time, $\bar{\theta}-\underline{\theta}>0$, so the constraints for $I R_{2}$ is strict true inequality.

\section{B. IR1 must be strict true equality, that is $s(\underline{r})=\underline{\theta} h(\underline{r})$}

By using reduction to absurdity, assumed $I R_{l}$ strict inequality is true, and based on the above Conclusions, $I R_{2}$ strict inequality is true. If the two constraint conditions make the inequalities true simultaneously, then the central government can raise $h(\bar{r})$ and $h(\underline{r})$ in equal quantity, assuming enough small amount of quantity $\varepsilon>0$ is raised, then constraint of $I R$ is not affected by any factors. For the constraint of $I R$, the strict inequality is still true because of raising extremely small quantity. Thus, no matter what kinds of local government, if the two inequalities constrained for $I R$ the is strict true at the same, that means the income of central government would raised which contradict with hypothesis of $E u_{l}$ maximizing, so when $I R_{2}$ is true inequalities, $I R_{l}$ must be equality true.

\section{The equality constrained for $\mathrm{IC}_{2}$ is true}

The reduction to absurdity still is used to prove, assume that the inequality constrained for $I R_{2}$ is strict true, if the reward raised by central government for $\bar{\theta}$ type of local government is $\varepsilon$, the equality of $I R_{l}$ is not affected, and the equality still is true. For the inequality of $I C_{2}$, it can be hypothesized:

$$
\begin{gathered}
\varepsilon=\{[\bar{\theta} h(\bar{r})-s(\bar{r})]-[\bar{\theta} h(\underline{r})-s(\underline{r})]\} / 2, \text { then } \\
\bar{\theta} h(\bar{r})-s(\bar{r})-\varepsilon=\{[\bar{\theta} h(\bar{r})-s(\bar{r})]+[\bar{\theta} h(\underline{r})-s(\underline{r})]\} / 2 \geq \bar{\theta} h(\underline{r})-s(\underline{r})
\end{gathered}
$$

Inequality of $I C_{2}$ is still true. That means the central government can increase the expected profit by raising the reward of $\bar{\theta}$ type of local government which does not accord with the hypothesis, so it can concluded that the equality constrained for $I C_{2}$ is true. 
D. Without considering the constraint condition for IC1, solve the planning problems of sub-constraint

It is concluded from above demonstration:

$$
\begin{aligned}
& I R_{1}: \underline{\theta} h(\underline{r})-s(\underline{r})=0 \\
& I C_{2}: \bar{\theta} h(\bar{r})-s(\bar{r})=\bar{\theta} h(\underline{r})-s(\underline{r}) \\
& s(\underline{r})=\underline{\theta} h(\underline{r}) \\
& s(\bar{r})=\bar{\theta} h(\bar{r})-(\bar{\theta}-\underline{\theta}) h(\underline{r})
\end{aligned}
$$

The above result is introduced into $I C_{l}$, it can prove that the constraint condition of $I C_{l}$ is true, so the solution for planning problems of sub-constraint is same with the solution for planning problems of original constraint.

\section{E. Solution scheme $\left(-\frac{r}{-}, \operatorname{and}(\bar{r}, \bar{T})\right.$}

The equality (8)and (9) are introduced into function for securities company' expected profit $E u_{1}$, then:

$$
\begin{aligned}
& E u_{1}=p(\bar{T}-\bar{h})+(1-p)(\underline{T}-\underline{h})= \\
& p[\bar{\theta} s(\bar{r})-h(\bar{r})-(\bar{\theta}-\underline{\theta}) s(\underline{r})]+(1-p)[\underline{\theta} s(\underline{r})-h(\underline{r})]
\end{aligned}
$$

When $E u_{1}$ reaches a maximum; its first partial derivatives shall be equal to 0 , then:

$$
\begin{aligned}
& \frac{\partial E u_{1}}{\partial \bar{r}}=0, \frac{\partial E u_{1}}{\partial \underline{r}}=0 . \\
& \text { Then: } \bar{\theta} s^{\prime}(\bar{r})=h^{\prime}(\bar{r}) \\
& (\underline{\theta}-p \bar{\theta}) s^{\prime}(\underline{r})=(1-p) h^{\prime} \underline{(r)}
\end{aligned}
$$

Because the nonlinear function $h(r)$ and $s(r)$ are the common knowledge for two parties, when the two functions are known, it can solve $\bar{r}$ and $\underline{r}$, and then solve $\bar{h}$ and $\underline{h}$ according with equality (10) and (11). In the last, central government confirm the reward system $(\underline{r}, \underline{h})$ and $(\bar{r}$, $\bar{h}$ )based on local government' judgment rate.

\section{ANALYSIS OF MODEL RESULT}

The central government can formulate the reward systems based on the derivation of the above formula to mobilize enthusiasm and initiative of local government on construct affordable housing. For local government, the construction of indemnificatory housing means lose of land-transferring fees which are regarded as mainly economic source. The construction of indemnificatory housing is non-profit, it is difficult to finance for entering into, which mainly come from the financing platform established and led by local government. Financing problem is the key factor for whether the local government completes the construction of affordable housing smoothly.

Facing many difficulties of financing and reducing fiscal revenue, how to enable local government to participate actively and to achieve the construction task smoothly have became the issues local government must deal with. Firstly, the objective of construction of affordable housing shall meet the conditions of all provinces and cities which require do it within capabilities and best meet housing needs of medium and lowincome population. Secondly, the reward provided for local government shall treat at different levels. It needs to offer certain financial supports for provinces and cities do well and offer policy support at other ways which ensure make up the local government' fiscal revenue, and that can also increase the initiative of local government on the construction of affordable housing.

\section{REFERENCES}

[1] Luo Xia, Wang Chonfeng, Study on Financing Channel Expansion for Our Country' Construction of Affordable Housing [J]. Foreign Investment in China. 2015(12). (In Chinese)

[2] Zhao Cunyan. Cost-Benefit Analysis for Local Government's Affordable Housing $[\mathrm{J}]$. Business Culture ( The First Fortnightly). 2016(05). (In Chinese)

[3] Hu Lianxia, Hu Wenye, Construction of Affordable Housing for Local Government [J]. Technical Information. 2015(06). (In Chinese)

[4] Chen Datao. Dynamic Mechanism Study on Construction of Affordable Housing under Residential Land' Dual System [J]. Zhejiang Academic Journal. 2016(04). (In Chinese) 